\title{
Automatic extraction of the curved midsagittal brain surface on MR images
}

\author{
Hugo J. Kuijf ${ }^{1}$, Max A. Viergever ${ }^{1}$, and Koen L. Vincken ${ }^{1}$ \\ Image Sciences Institute, University Medical Center Utrecht, the Netherlands
}

\begin{abstract}
Many methods exist for the automatic extraction of the midsagittal plane from neuroimages, assuming bilateral symmetry. However, this assumption is incorrect owing to brain torque and the possible presence of pathology. In this paper, a method for extracting the curved midsagittal surface from brain images is presented.

First, the method localizes the interhemispheric fissure with an existing technique for midsagittal plane extraction. Next, the plane is modelled as a bicubic spline and the configuration of the control points is optimized to obtain the midsagittal surface.

The midsagittal surface results in a better segmentation of the cerebral hemispheres. Not only is the result visually more appealing, the absolute volume of misclassified tissue decreases significantly.
\end{abstract}

\section{Introduction}

Bilateral symmetry is an important concept in biology and many animal species, including humans. Our appearance exhibits bilateral symmetry and some organs in our body come in symmetrical pairs, for example our brain. The cerebrum is divided into two hemispheres, separated by the interhemispheric fissure (IF). Comparison of the two hemispheres and detection of differences has been a topic of discussion for many years. Besides the lateralization of brain function, anatomical differences can suggest the presence of pathology (like a brain mass or tumour), indicate schizophrenia [1], or various other diseases.

The midsagittal plane is a geometric plane that separates the two hemispheres and coincides with the IF. In the past years, multiple methods have been published to extract the midsagittal plane from neuroimages. Assuming bilateral symmetry, most of the methods work by optimizing a symmetry metric between the neuroimage and a reflected version of itself.

However, the human brain has no perfect bilateral symmetry. The left occipital and right frontal lobe are larger than their counterparts in the other hemisphere are, which is known as brain torque. Besides brain torque, the presence of brain masses could induce asymmetries in the cerebrum. Existing techniques to extract the midsagittal plane from neuroimages do not take these asymmetries into account and might therefore fail to correctly segment the two hemispheres.

The midsagittal surface is a curved surface following the IF. In the presence of asymmetries, either owing to natural variation or pathology, the midsagittal 
surface will correctly segment the two hemispheres, whereas a midsagittal plane would intersect or misclassify some brain tissue. It is therefore likely that the midsagittal surface will result in more accurate analysis of interhemispherical differences.

In the present study, a novel method for extracting the curved midsagittal surface will be presented, based on an existing method for extracting the midsagittal plane.

\section{Methods and Materials}

\subsection{Participants \& MRI}

A total of 50 consecutive participants (mean age: 59 years, sd: 10 years) from the SMART study [2] have been included for evaluation of the method. The SMART study was approved by the Medical Ethics Committee and written informed consent was given by all participants.

MRI acquisition was performed on a $1.5 \mathrm{~T}$ whole-body system (Gyroscan ACS-NT, Philips Medical Systems, Best, the Netherlands). The protocol included, among others, a transversal T1-weighted gradient-echo sequence (repetition time (TR)/echo time (TE): 235/2 ms); a transversal T2-weighted fluidattenuated inversion recovery (FLAIR) (TR/TE/inversion time (TI): 6000/100/ $2000 \mathrm{~ms}$ ), and a transversal inversion recovery (IR) (TR/TE/TI: 2900/22/410 $\mathrm{ms}$ ), all with a reconstructed voxel size of $0.9 \times 0.9 \times 4.0 \mathrm{~mm}$.

For extraction of the midsagittal plane and surface, the T1-weighted sequence was used.

\section{$2.2 \quad$ Midsagittal plane}

Many methods exist for the automatic extraction of the midsagittal plane, which can be roughly divided into two categories: symmetry-based methods (e.g. [310]) and fissure-based methods ([11-13]). Symmetry-based methods work with the implicit assumption that the brain possesses bilateral symmetry. These methods try to align the image with a reflected version of itself, while optimizing a symmetry-metric. However, due to the asymmetric nature of the brain, these techniques sometimes fail.

Fissure-based methods try to detect the IF, based on its distinctive characteristics visible in the image. With imaging modalities as CT and MRI, the cerebrospinal fluid (CSF) located in the IF gives a high visual contrast with the surrounding gray and white matter of both hemispheres. This contrast is clearly visible in Figures 1(a), (d), and (g), and can be used to extract the midsagittal plane and surface. A fissure-based method for extracting the midsagittal plane is described by Volkau et al. [12] and Nowinski et al. [13] and was used in the present study. The approach of this method, as will be explained below, allows for extension to extract the midsagittal surface and thus formed an ideal candidate for the present study. 
First, two reference planes were taken $2 \mathrm{~cm}$ apart from the central sagittal slice of the image. As the method assumes that the brain is approximately located in the centre of the image, these reference planes consist mostly of gray and white matter. A single probability distribution of the gray values present in the two references slices was created.

Next, all slices in-between the two reference slices were inspected. For each slice, a probability distribution of the gray values was created. The KullbackLeibler (KL) divergence was computed using the reference probability distribution and the probability distribution of the current slice. This resulted in a measure of the difference between the two probability distributions (the KLvalue). As the reference slices contains mostly gray and white matter and the IF contains mostly CSF, the slice containing (a large part of) the IF would result in a relatively large KL-value.

The sagittal slice with the largest KL-value was taken as an initial guess for the MSP. As the brain can be rotated, the IF will not always perfectly align with a sagittal slice in the image. Therefore, three random corner points of the MSP were taken and shifted along the left/right axis of the scan. The location of these three corner points could be optimized in terms of the KL-value. For each new location, the KL-value was computed and the rotated slice with the largest difference to the reference distribution was taken as the final MSP. This process is summarized in Figure 1.

\subsection{Midsagittal surface}

The midsagittal plane computed in the previous section was used to initialize the method for extracting the midsagittal surface. The surface was represented as a bicubic spline, as implemented in ALGLIB [14]. Control points for the spline were placed in a regular $m \times n$ grid on the computed MSP, having $m$ be the number of control points in the anterior-posterior direction and $n$ in the headfeet direction. The values of $m$ and $n$ were user-defined. An example is shown in Figure 2(a)

An optimization method was used to determine the optimal configuration of the control points. The control points could only be moved along the left/right axis of the scan during optimization. The Kullback-Leibler's divergence was used as a cost function that needed to be maximized. It used the previously computed reference probability distribution and generated a probability distribution of the bicubic spline during optimization.

A limited-memory Broyden-Fletcher-Goldfarb-Shanno quasi-newton method (L-BFGS), as implemented in the dlib $\mathrm{C}++$ library [15], was used to determine the direction of the search. This method required gradient information of the cost function to be optimized, which was numerically approximated. The step size of each control point in each iteration was scaled with the gradient at each control point, allowing subvoxel accuracy in the configuration. The optimization method was terminated when the cost function converged: two consecutive optimization steps had a difference in KL-value of $1 \times 10^{-5}$ or less. An example of this procedure can be seen in Figure 2. 


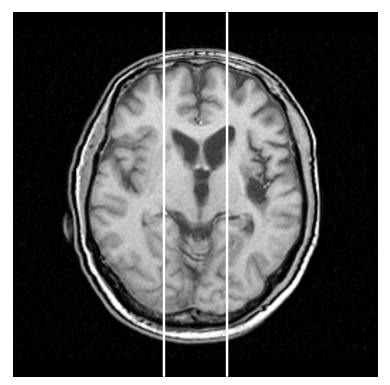

(a)

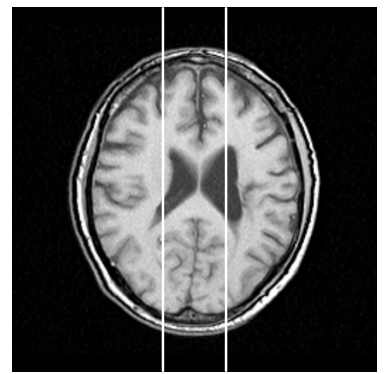

(d)

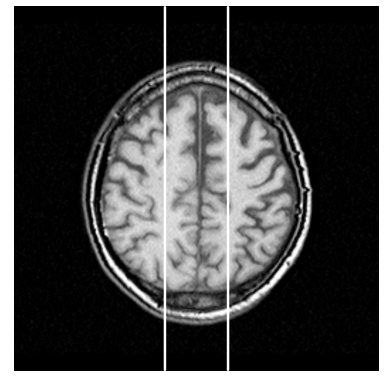

(g)

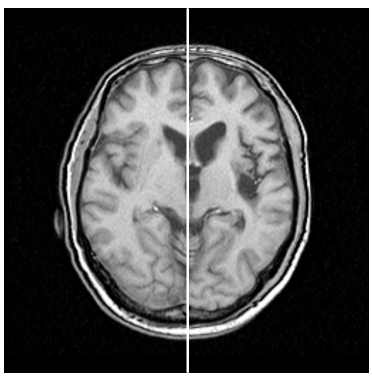

(b)

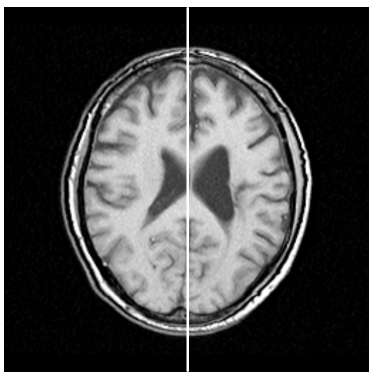

(e)

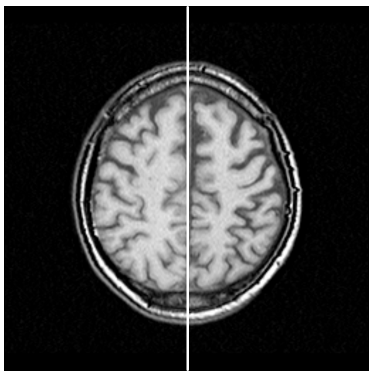

(h)

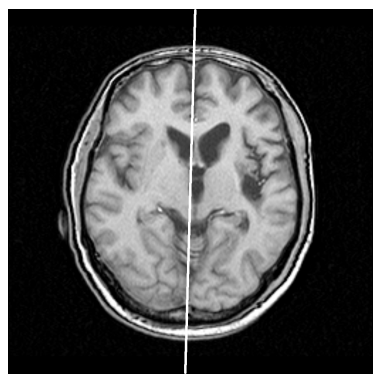

(c)

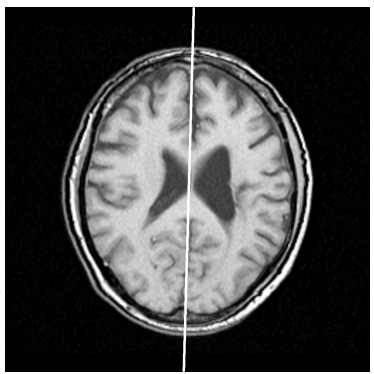

(f)

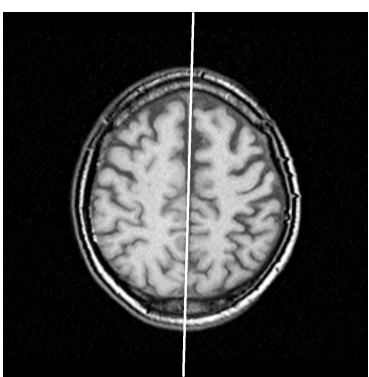

(i)

Fig. 1. Extraction of the midsagittal plane shown on three slices taken from one scan. Left: lines indicate the two initial reference planes. Middle: line indicates the sagittal slice with the largest KL-value. Right: line indicates the midsagittal plane.

\subsection{Experiments and Validation}

The quality of the midsagittal plane and surface extraction was evaluated visually and quantitatively in the cerebrum, ignoring the cerebellum. The cerebellum was ignored, since a left/right segmentation is ambiguous and ill-defined. This is commonly done in segmentation algorithms. [16] For the quantitative validation, the brain tissue volume in the cerebrum that was classified as either left or right was assessed automatically and compared to a ground truth. First, reasonable settings for $m$ and $n$ were determined heuristically on a smaller subset of par- 


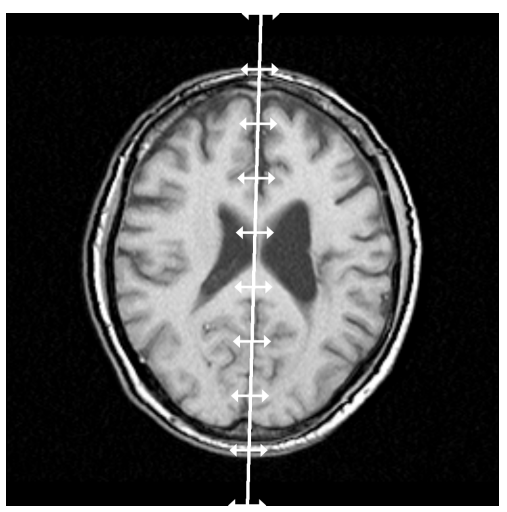

(a)

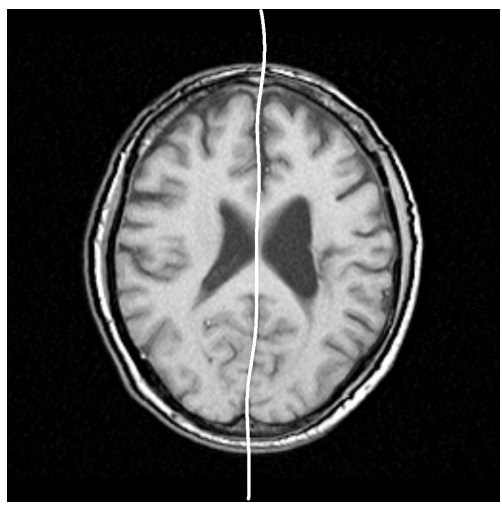

(b)

Fig. 2. Left: Figure 1(f) is shown with the control points for the optimization. The arrows indicate the direction in which the control points could move. Right: Optimal configuration according to the KL-divergence is shown. The error that the midsagittal plane made at the left occipital lobe was corrected by the computed surface.

ticipants. The influence of these parameters was assessed visually and fixed for the quantitative analysis.

Second, a gray and white matter segmentation was obtained with a probabilistic k-Nearest Neighbour classification segmentation method. This method used the T1-weighted, IR, and FLAIR sequences, as described by Anbeek et al. [17]

Using the MNI152 template[18,19], a ground truth left/right segmentation was created. For this template, a true left/right atlas of the cerebrum is available. By computing a deformable registration of the MNI152 template to the T1-weighted scan, the left/right atlas could be propagated to the gray and white matter segmentation. Registrations were computed with elastix [20], with registration parameters taken from Van der Lijn et al. [21] The quality of the registration was assessed visually by an experienced observer and all registrations were considered accurate.

\section{Results}

Values for $m$ and $n$ were set at 10 and 5. Lower values were unable to capture the curvature of the IF and higher values resulted in overfitting of the spline.

The results of the extraction of the midsagittal plane were visually inspected for correctness. In all cases, the midsagittal plane was found correctly and aligned with the IF, as was also previously reported by Volkau and Nowinski. $[12,13]$ However, small errors were made by the method, mostly at the left occipital lobe (as visible in Figure 1) and the right frontal lobe. This was to be expected, owing to the possible presence of brain torque. 
The midsagittal surface showed a visually more appealing result than the midsagittal plane. The surface followed the interhemispheric fissure at locations where the midsagittal plane would cut through tissue. An example of this was shown in Figure 2(b) and more results are shown in Figure 3.

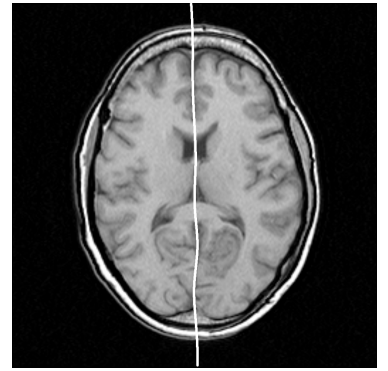

(a)

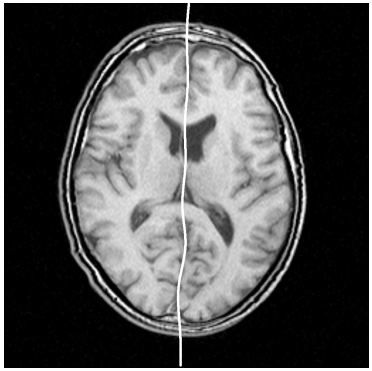

(b)

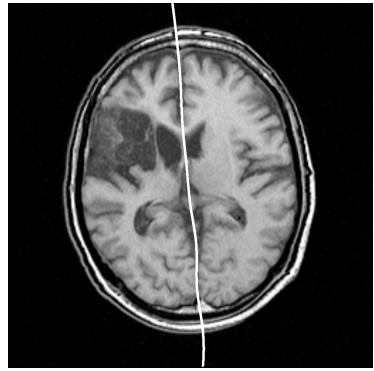

(c)

Fig. 3. Example results of the method. (b) The midsagittal surface was sometimes fitted through the lateral ventricles. (c) Asymmetries in the brain do not influence the results, as would be the case for symmetry-based methods.

For the quantitative validation, the absolute volume of tissue in the cerebrum that was misclassified (i.e. classified as left where it should be right, or vice versa) was assessed automatically. In the ideal case, this error would be zero. The average error (mean $\pm \mathrm{sd}$ ) of the midsagittal plane was $1.06 \pm 0.89 \mathrm{ml}$ and the average error of the midsagittal surface was $0.59 \pm 0.63 \mathrm{ml}$. The difference between the midsagittal plane and the midsagittal surface was statistically significant, using a one-sided, paired, Student's t-test, with a $p$-value of $1.0 \times 10^{-6}$.

Computation time of the midsagittal plane was approximately 2 seconds. Depending on the number of iterations required, the computation of the midsagittal surface was 2 to 10 seconds.

\section{Discussion}

The implementation of the midsagittal surface shows a clear improvement over the midsagittal plane. Not only does the midsagittal surface show a visually more correct and appealing result, the improvement in the absolute volume of misclassified tissue is statistically significant. Besides the statistical significance, the absolute reduction in the error with $0.5 \mathrm{ml}$ on average is relevant in many applications. Although a volume $0.5 \mathrm{ml}$ is relatively small compared to the whole brain volume, it is a considerable amount of tissue in the vicinity of the IF. Next to the average reduction in error, the standard deviation of the error also decreases. This indicates that the midsagittal surface gives a more robust estimate of the left-right segmentation than the midsagittal plane does. 
An error sometimes made by the midsagittal surface is found at the location of the lateral ventricles. During optimization, the cost function will try to avoid the septum pellucidum, the membrane separating the lateral ventricles, and fit the spline through the CSF-filled ventricles. This will give a more optimal solution in terms of KL-value, although it is not the most logical separation at that location. However, it has no influence on the left/right segmentation of the tissue in the cerebrum. An example was shown in Figure 3(b).

The quantitative validation of the method required an atlas-registration of the MNI152 template to each individual image. By doing this, the quality of the ground truth depends on the quality of the registration. Thorough inspection of the registration results by an experienced observer did not reveal any errors in the left-right segmentation of the cerebrum generated by the registration.

Of course, one could argue that having a left-right segmentation available by means of registration with the MNI152 template already solves the problem of extracting the midsagittal surface. However, the registration of the MNI152 template to the scans required 7 minutes per scan. The computation of the midsagittal surface required, at most, 12 seconds, making an expensive atlas registration superfluous.

The method works without adaptation on other image contrasts, such as FLAIR and IR, and higher field strengths, such as 3.0T or 7.0T. The only prerequisite for the method is a visible contrast between the interhemispheric fissure and surrounding tissue. The method can be applied to other imaging modalities, such as CT, as well. [22]

Besides segmentation of the cerebrum into the left and right hemispheres, there is a possibility to use this method for the detection of midline shift. Techniques for this application have been published before [23], using the midsagittal plane and a Bézier curve. The midsagittal surface could be used instead, without the limited degrees of freedom of a Bézier curve.

\section{References}

1. Crow, T.: Schizophrenia as an anomaly of cerebral asymmetriy. Imaging of the Brain in Psychiatry and Related Fields (1993) 1-17

2. Anonymous

3. Junck, L., Moen, J.G., Hutchins, G.D., Brown, M.B., Kuhl, D.E.: Correlation methods for the centering, rotation, and alignment of functional brain images. Journal of Nuclear Medicine 31(7) (1990) 1220-1226

4. Minoshima, S., Berger, K.L., Lee, K.S., Mintun, M.A.: An automated method for rotational correction and centering of three-dimensional functional brain images. Journal of Nuclear Medicine 33(8) (1992) 1579-1585

5. Ardekani, B.A., Kershaw, J., Braun, M., Kanno, I.: Automatic detection of the mid-sagittal plane in 3-d brain images. IEEE Transactions on Medical Imaging 16(6) (12 1997) 947-952

6. Smith, S., Jenkinson, M.: Accurate robust symmetry estimation. In Taylor, C., Colchester, A., eds.: Medical Image Computing and Computer-Assisted Intervention - MICCAI'99. Volume 1679 of Lecture Notes in Computer Science. Springer Berlin / Heidelberg (1999) 308-317 
7. Liu, Y., Collins, R., Rothfus, W.E.: Robust midsagittal plane extraction from normal and pathological 3d neuroradiology images. IEEE Transactions on Medical Imaging 20(1) (March 2001) 175 - 192

8. Prima, S., Ourselin, S., Ayache, N.: Computation of the mid-sagittal plane in 3-d brain images. IEEE Transactions on Medical Imaging 21(2) (2 2002) 122-138

9. $\mathrm{Hu}, \mathrm{Q}$., Nowinski, W.L.: A rapid algorithm for robust and automatic extraction of the midsagittal plane of the human cerebrum from neuroimages based on local symmetry and outlier removal. NeuroImage 20(4) (2003) 2153 - 2165

10. Tuzikov, A.V., Colliot, O., Bloch, I.: Evaluation of the symmetry plane in $3 \mathrm{~d} \mathrm{mr}$ brain images. Pattern Recognition Letters 24(14) (2003) 2219 - 2233

11. Brummer, M.E.: Hough transform detection of the longitudinal fissure in tomographic head images. IEEE Transactions on Medical Imaging 10(1) (3 1991) 74-81

12. Volkau, I., Prakash, K.B., Ananthasubramaniam, A., Aziz, A., Nowinski, W.L.: Extraction of the midsagittal plane from morphological neuroimages using the kullback-leibler's measure. Medical Image Analysis 10(6) (2006) 863 - 874

13. Nowinski, W.L., Prakash, B., Volkau, I., Ananthasubramaniam, A., Beauchamp Jr, N.J.: Rapid and automatic calculation of the midsagittal plane in magnetic resonance diffusion and perfusion images. Academic Radiology 13(5) (2006) 652 $-663$

14. Bochkanov, S., Bystritsky, V.: Alglib (www.alglib.net)

15. King, D.E.: Dlib c++ library (www.dlib.net)

16. Liang, L., Rehm, K., Woods, R.P., Rottenberg, D.A.: Automatic segmentation of left and right cerebral hemispheres from mri brain volumes using the graph cuts algorithm. NeuroImage 34(3) (2007) 1160 - 1170

17. Anbeek, P., Vincken, K.L., van Bochove, G.S., van Osch, M.J., van der Grond, J.: Probabilistic segmentation of brain tissue in mr imaging. NeuroImage 27(4) (2005) $795-804$

18. Fonov, V., Evans, A., McKinstry, R., Almli, C., Collins, D.: Unbiased nonlinear average age-appropriate brain templates from birth to adulthood. NeuroImage 47, Supplement 1 (2009) S102 OHBM 2009 Annual Meeting.

19. Fonov, V., Evans, A.C., Botteron, K., Almli, C.R., McKinstry, R.C., Collins, D.L.: Unbiased average age-appropriate atlases for pediatric studies. NeuroImage 54(1) (2011) $313-327$

20. Klein, S., Staring, M., Murphy, K., Viergever, M., Pluim, J.: elastix: A toolbox for intensity-based medical image registration. Medical Imaging, IEEE Transactions on 29(1) (jan. 2010) $196-205$

21. van der Lijn, F., de Bruijne, M., Hoogendam, Y., Klein, S., Hameeteman, R., Breteler, M., Niessen, W.: Cerebellum segmentation in mri using atlas registration and local multi-scale image descriptors. In: Biomedical Imaging: From Nano to Macro, 2009. ISBI '09. IEEE International Symposium on. (2009) $221-224$

22. Puspitasari, F., Volkau, I., Ambrosius, W., Nowinski, W.: Robust calculation of the midsagittal plane in ct scans using the kullbackleiblers measure. International Journal of Computer Assisted Radiology and Surgery 4 (2009) 535-547

23. Liao, C.C., Xiao, F., Wong, J.M., Chiang, I.J.: Automatic recognition of midline shift on brain ct images. Computers in Biology and Medicine 40(3) (2010) 331 339 